\title{
A DIRECT TOPOLOGICAL DEFINITION OF THE FULLER INDEX FOR LOCAL SEMIFLOWS
}

\author{
Christian C. Fenske
}

\begin{abstract}
We define an index of Fuller type counting the periodic orbits of a local topological semiflow on ANR spaces avoiding smoothness assumptions and approximation processes
\end{abstract}

\section{Introduction}

Fuller in his classical article [5] defined an index counting isolated sets of periodic orbits of a smooth vector field $X$ on a manifold $M$. A major step in the argument consists in approximating $X$ by a vector field that has only finitely many periodic orbits all of which are hyperbolic. Here, we will not assume any differentiability - we will just consider a local semiflow. In this situation there is no conceivable way to approximate the semiflow by a semiflow having only finitely many periodic orbits. In [4] we proposed a complicated approximation process which approximates the semiflow by a parametrized mapping which respects the flow structure on sufficiently many periodic orbits. Here, it is our objective to define the index entirely in terms of the semiflow. Our starting point is the following expression [2]

$$
\bar{H}^{n}(X \times X, X \times X \backslash \Delta) \stackrel{g^{*}}{\longrightarrow} \bar{H}^{n}(\Omega, \Omega \backslash P) \cong \bar{H}_{1} P \stackrel{i_{*}}{\longrightarrow} H_{1} \Omega .
$$

2000 Mathematics Subject Classification. Primary: 37C25; Secondary: 54H20, 55M25.

Key words and phrases. Fuller index, periodic orbit, local semiflow. 
Here, $X$ is open in $\mathbb{R}^{n}, \Omega$ is an open subset of $\mathbb{R}^{n+1}, \Phi$ is a local semiflow on $X$ with $\bar{\Omega} \subset \mathcal{D}(\Phi)$ (the domain of definition of $\Phi), P:=\left\{(x, t) \in \bar{\Omega} \mid \phi_{t} x=x\right\}$, $g: \Omega \rightarrow X$ is the mapping $(x, t) \mapsto\left(x, \phi_{t} x\right), \bar{H}^{*}$ is Alexander-Spanier cohomology, $\cong$ is Poincaré duality, $\bar{H}_{*}$ is the homology of the complex with $C_{q}(X, A ; G)=$ $\operatorname{Hom}\left(C_{c}^{q}(X, A ; G), G\right)$ where $C_{c}^{q}$ denotes Alexander-Spanier cochains with compact support, and $i: P \rightarrow \Omega$ is the inclusion. By $H_{*}^{s}$ we denote singular homology, and we just write $H_{*}$ or $H^{*}$ when there is no difference between the (co-)homology theories. The homological index $I(X, \Phi, \Omega)$ then is defined to be the image of the diagonal class $\tau_{X} \in H^{n}(X \times X, X \times X \backslash \Delta)$ in the above diagram. Of course, there are some assumptions, viz.

(H'1) $P$ is a compact subset of $\Omega$.

(H'2) There is a $t_{0} \geq 0$ such that $\phi_{t_{0}}$ is compact and $\operatorname{pr}_{2} P \subset\left(t_{0}, \infty\right)$.

(H'3) $\Phi$ has a compact attractor.

Now it would be straightforward to define a numerical index if one could show that $I(X, \Phi, \Omega)$ can be represented as a cycle $\sum_{i=1}^{r} c_{i} \gamma_{i}$ where $\gamma_{1}, \ldots, \gamma_{r}$ are periodic orbits of $\Phi$ because then we would define the numerical index as $i(X, \Phi, \Omega)=\sum_{i=1}^{r} c_{i} / m_{i}$ where $m_{i}$ is the multiplicity of $\gamma_{i}$. Since I do not see how to prove this assertion (although there is some evidence in favour of this conjecture) let us observe that it would be sufficient to find a canonical projection from $\bar{H}_{1} P$ to the subgroup generated by the periodic orbits. Note that there is an operation of $S^{1}$ on $P, T: S^{1} \times P \rightarrow P$, defined by $T(\exp (2 \pi \mathrm{i} \theta),(x, t))=\phi_{\theta t} x$ where $0 \leq \theta \leq 1$. Now (ignoring difficulties) we could hope to replace $\bar{H}_{1}\left(S^{1} \times P\right)$ by $\left(\bar{H}_{0} S^{1} \otimes \bar{H}_{1} P\right) \oplus\left(\bar{H}_{1} S^{1} \otimes \bar{H}_{0} P\right)$. So we should be looking for a mapping $S: \bar{H}_{1} P \rightarrow \bar{H}_{1} S^{1} \otimes \bar{H}_{0} P \cong \bar{H}_{0} P$. Applying $T_{*}$ we will end up in the subgroup generated by the periodic orbits. The actual construction will be somewhat different, but this is the basic idea. There is, however, one more task which has to be solved. If $H_{1} \Omega=0$ then $I(X, \Phi, \Omega)=0$ so the topological index does not give any information. Fuller (op. cit.) circumvented this difficulty by an ingenious configuration-space argument. Call $X^{(k)}$ the subset of those $x_{1}, \ldots, x_{k} \in X^{k}$ consisting of $k$ different points. The cyclic group $\mathbb{Z}_{k}$ acts on $X^{(k)}$ (the action being generated by the shift $\left.\left(x_{1}, \ldots, x_{k}\right) \mapsto\left(x_{2}, \ldots, x_{k}, x_{1}\right)\right)$. Call $X_{k}=X^{(k)} / \mathbb{Z}_{k}$. If $\Phi$ is a local flow on $X$ then $\left(x_{1}, \ldots, x_{k}, t\right) \mapsto\left(\phi_{t} x_{1}, \ldots, \phi_{t} x_{k}\right)$ defines a local flow on $X^{(k)}$ which yields a local flow on $X_{k}$. If, however, $\Phi$ is just a semiflow then $\phi_{t} x_{1}, \ldots, \phi_{t} x_{k}$ need not be $k$ different points for $\left(x_{1}, \ldots, x_{k}\right) \in$ $X^{(k)}$. In [3] we have explained how to get around this difficulty in the case of a local semiflow.

Granting all this, there remains, however, still a drawback in our approach: the introduction of the configuration spaces $X_{k}$ forces us to use coefficients in $\mathbb{Z}_{k}$ (and not in $\mathbb{Q}$ as would be appropriate), and I have not been able to compare the indices for different values of $k$. 


\section{The setting of the problem}

We assume that $X$ is an ANR, $\Omega \subset X \times[0, \infty)$ is open, $\Phi$ is a local semiflow with $\bar{\Omega} \subset \mathcal{D}(\Phi)$ which satisfies (H'1)-(H'3). In this situation, it is well-known that $\Phi$ has a uniform compact attractor (i.e. a compact attractor with arbitrarily small invariant neighbourhoods). So by (H'2) there is an invariant neighbourhood $U$ such that $\operatorname{cl} \phi_{t_{0}}(U)$ is a compact subset of $U$. So we may replace $\Omega$ by a smaller open set $\Omega^{\prime} \supset P$ such that $\operatorname{cl} \Phi\left(\Omega^{\prime}\right)$ is compact, i.e. instead of (H'1)-(H'3) we may assume

(H1) $P$ is a compact subset of $\Omega$, and

$(\mathrm{H} 2) \operatorname{cl} \Phi(\Omega)$ is compact.

Definition. We say that an index for local semiflows is defined if for all triplets $(X, \Phi, \Omega)$ satisfying $(\mathrm{H} 1)$ and $(\mathrm{H} 2)$ and each sufficiently large prime $k$ we may define an element $i_{k}(X, \Phi, \Omega) \in \mathbb{Z}_{k}$ such that

(A) $i_{k}$ is additive with respect to $\Omega$, i.e. if $\Omega_{1}, \Omega_{2} \subset \Omega$ are open such that $P \cap\left(\bar{\Omega} \backslash\left(\Omega_{1} \cup \Omega_{2}\right)\right)=\emptyset$ then $i_{k}(X, \Phi, \Omega)=i_{k}\left(X, \Phi, \Omega_{1}\right)+i_{k}\left(X, \Phi, \Omega_{2}\right)$.

(H) ( $i_{k}$ is homotopy-invariant) If $\Omega \subset X \times[0, \infty) \times[0,1]$ is open and if we have a continuous map

$$
\Phi: \bar{\Omega} \rightarrow X, \quad(x, t, \lambda) \mapsto \Phi^{\lambda}(x, t)
$$

such that $\operatorname{cl} \Phi(\Omega)$ is compact and that with $\Omega^{\lambda}:=\Omega \cap((X \times[0, \infty)) \times\{\lambda\})$ we have that $\Phi^{\lambda}$ is a local semiflow with $\left(X, \Phi^{\lambda}, \Omega^{\lambda}\right)$ satisfying (H1) and (H2) then $i_{k}\left(X, \Phi^{0}, \Omega^{0}\right)=i_{k}\left(X, \Phi^{1}, \Omega^{1}\right)$ for all sufficiently large primes.

(N) The index is normalized: $i_{k}(X, \Phi, \Omega)$ is the element in $\mathbb{Z}_{k}$ corresponding to $\iota / m \in \mathbb{Q}$ if $P$ consists of a single periodic orbit $\gamma$ of multiplicity $m$ and $\iota$ is the fixed point index of the Poincaré mapping with respect to $\gamma$.

Two remarks are in order. In $(\mathrm{N})$ we mean that $i_{k}(X, \Phi, \Omega)$ solves the equation $m x=\iota$ in $\mathbb{Z}_{k}$. Secondly, we comment on the definition of the Poincaré mapping. Let $\Phi$ be a local semiflow on a metric space $(X, d)$ and $x$ a periodic point with minimal period $p$. Then $\gamma:=\Phi(\{x\} \times[0, p])$ is called the periodic orbit of $x$. (Here and in what follows we will write $|\gamma|:=\gamma([0, p])$. Moreover, for $x \in|\gamma|$, we denote the minimal period of $x$ by $p(x)$ or equivalently by $p(\gamma)$.) Choose a neighbourhood $\mathcal{O}$ of $|\gamma|$ such that all $y \in \mathcal{O}$ have lifetime at least $4 p$. Define $\psi: \mathcal{O} \times(-p, 2 p) \rightarrow[0, \infty)$ by

$$
\psi(y, t)=\int_{t}^{t+p / 4} d\left(x, \phi_{p+s} y\right) d s .
$$

In [1] it is proved that there is a $\delta>0$ and a $\rho<\delta$ such that with $S:=\{y \in$ $B(x ; \delta) \mid \psi(x, 0)=\psi(y, 0)\}$ and $\Sigma:=S \cap \bar{B}(x ; \rho)$ we have that the mapping $\tau: \Sigma \rightarrow(0, \infty)$ with $\tau(y)=\inf \left\{t>0 \mid \phi_{t} y \in S\right\}$ is defined and continuous. Then 
there is a neighbourhood $U$ of $|\gamma|$ such that the mapping with $\eta(y):=\inf \{t>$ $\left.0 \mid \phi_{t} y \in S\right\}$ is defined and continuous on $U \backslash S$.

Let $B$ be a small ball centered at $x$ and $y \in B$. Let $\sigma(y)=\tau(y)$ if $y \in S$, $\sigma(y)=\eta(y)$ if $\eta(y)>3 p / 4$, and $\sigma(y)=\eta(y)+\tau\left(\phi_{\eta(y)} y\right)$ if $\eta(y)<p / 4$. Let $\tau_{1}(y):=\sigma(y)$ and let $\tau_{j+1}(y):=\tau_{j}(y)+\tau\left(\phi_{\tau_{j}(y)} y\right)$. The Poincaré map $\pi: B \rightarrow S$ is then defined by $\pi(y)=\phi_{\tau_{m}(y)} y$.

In this situation, let us fix some terminology. Let $t_{+}>\max \{p(x) \mid(x, t) \in P\}$ be fixed. A pair $T^{\prime} \subset T$ of open neighbourhoods of $|\gamma|$ is called a tube around $\gamma$ and $\Sigma \subset T$ is called a section at $x$ if

- all points $y \in T^{\prime}$ have lifetime at least $t_{+}$and $\Phi\left(\{y\} \times\left[0, t_{+}\right]\right) \subset T$,

- for each $y \in \Sigma^{\prime}:=\Sigma \cap T^{\prime}$ we have that $\tau(y)=\inf \left\{t>0 \mid \phi_{t} y \in \Sigma\right\}$ is defined and defines a continuous mapping $\tau: \Sigma^{\prime} \rightarrow(0, \infty)$.

We now proceed as follows. We start by assuming that $X=M$ is an orientable manifold. First we recall the construction of nonbounding cycles. Next, we turn to the definition of the index. We will then explain how to extend the definition to the case of simplicial complexes and to the nonorientable case. Finally, we will deal with local semiflows on ANR spaces (cf. [7] for facts about ANR).

\section{Construction of nonbounding cycles}

Let $M$ be an $n$-dimensional orientable manifold, $\Omega \subset M \times(0, \infty)$ open, $\Phi: \mathcal{D}(\Omega) \rightarrow M$ a local semiflow such that $\bar{\Omega} \subset \mathcal{D}(\Phi)$.

Assume that $P:=\left\{(x, t) \in \bar{\Omega} \mid \phi_{t} x=x\right\}$ is a compact subset of $\Omega$. Let $\mu$ be the largest multiplicity occurring in $P$ and let $k>\mu^{2}$ be a natural number such that all prime divisors of $k$ are larger than $\mu^{2}$.

Denote by $M^{(k)}$ the set of those $\left(x_{1}, \ldots, x_{k}\right) \in M^{k}$ for which $x_{i} \neq x_{j}$ whenever $1 \leq i<j \leq k . M^{(k)}$ is again an orientable manifold. The cyclic group $\mathbb{Z}_{k}$ operates on $M^{(k)}$ by translations the generator being $\zeta_{1}:\left(x_{1}, x_{2}, \ldots, x_{k}\right) \mapsto$ $\left(x_{2}, \ldots, x_{k}, x_{1}\right)$. We denote the action of $\zeta \in \mathbb{Z}_{k}$ on $\xi \in M^{(k)}$ by $(\zeta, \xi) \mapsto \zeta \cdot \xi$. Call $M_{k}:=M^{(k)} / \mathbb{Z}_{k}$. Then $q_{k}: M^{(k)} \rightarrow M_{k}$ is a regular $k$-sheeted covering with group of covering transformations $\mathbb{Z}_{k}$, and $M^{(k)}$ and $M_{k}$ are manifolds.

Let $Q_{k}: M^{(k)} \times[0, \infty) \rightarrow M_{k} \times[0, \infty)$ be defined by $Q_{k}(\xi, t)=\left(q_{k}(\xi), t\right)$. For $(x, t) \in \mathcal{D}(\Phi)$ let $g_{k}(x, t)=\left(x, \phi_{t / k} x, \ldots, \phi_{(k-1) t / k} x\right)$ and $G_{k}(x, t):=\mathbb{Z}_{k} \cdot g_{k}(x, t)$.

LemmA. There are open sets $P \subset \Omega^{\prime \prime} \subset \bar{\Omega}^{\prime \prime} \subset \Omega^{\prime} \subset \bar{\Omega}^{\prime} \subset \Omega$ and a $\delta>0$ such that

(i) $\left(\phi_{\tau} x, t\right) \in \Omega$ whenever $(x, t) \in \Omega^{\prime}$ and $0 \leq \tau \leq 2 t$.

(ii) $g_{k}\left(\phi_{\tau} x, t\right) \in M^{(k)}$ whenever $(x, t) \in \Omega^{\prime}$ and $0 \leq \tau \leq 2 t$.

(iii) $q_{k} g_{k}(x, t)=q_{k} g_{k}\left(\phi_{s} x, t\right)$ for $(x, t) \in \Omega^{\prime}$ and $|s-t / k|<\delta$ if and only if $(x, t) \in P$ and $s=t / k$. 
(iv) $\left(\phi_{\tau} x, t\right) \in \Omega^{\prime}$ whenever $(x, t) \in \Omega^{\prime \prime}$ and $0 \leq \tau \leq t$.

This is proved in [3] in case $k>\mu$ is a prime. An inspection of the proof, however, shows that it is sufficient to assume that all prime divisors of $k$ are larger than $\mu$.

Now let $\mathcal{O}^{(k)}:=\left\{\left(\zeta \cdot g_{k}(x, t), t, s\right)\left|(x, t) \in \Omega^{\prime \prime}, \zeta \in \mathbb{Z}_{k},\right| s-t / k \mid<\delta\right\}$, $Z^{(k)}:=\left\{\left(\zeta \cdot g_{k}(x, t), t\right) \mid(x, t) \in \Omega^{\prime}, \zeta \in \mathbb{Z}_{k}\right\} . Z^{(k)}$ is a manifold. Let $(x, t) \in \Omega^{\prime \prime}$ and $\zeta \in \mathbb{Z}_{k}$ and choose a coordinate patch $U \ni x$ in $\Omega^{\prime \prime}$ such that $\phi_{t / k}(U) \cap U=\emptyset$. Then $\zeta \cdot g_{k}$ maps $U$ homeomorphically onto $\zeta \cdot g_{k}(U) . \mathcal{O}^{(k)}$ is an open subset of $Z^{(k)} \times(0, \infty)$, hence a manifold. (In [3] there is a flaw in the argument. In that article I have used $g_{k}(x, t)$ rather than the $\mathbb{Z}_{k}$-orbit of $g_{k}(x, t)$, but then the preimage of a point in $Z_{k}$ under $q_{k}$ consists of a single point unless the point is on a periodic orbit.)

Define a local semiflow $\Psi^{(k)}$ on $Z^{(k)}$ by

$$
\psi_{\tau}^{(k)}\left(\zeta \cdot g_{k}(x, t), t\right):=\left(\zeta \cdot g_{k}\left(\phi_{\tau} x, t\right), t\right)
$$

Let

$$
\begin{aligned}
\mathcal{O}_{k} & :=\left\{\left(q_{k} g_{k}(x, t), t, s\right)\left|(x, t) \in \Omega^{\prime \prime},\right| s-t / k \mid<\delta\right\}, \\
Z_{k} & :=\left\{\left(q_{k} g_{k}(x, t), t\right) \mid(x, t) \in \Omega^{\prime}\right\} .
\end{aligned}
$$

There is a local semiflow $\Psi^{k}$ on $Z_{k}$ defined by

$$
\psi_{\tau}^{k}\left(q_{k} g_{k}(x, t), t\right)=\left(q_{k} g_{k}\left(\phi_{\tau} x, t\right), t\right) .
$$

Obviously, $q_{k}: M^{(k)} \rightarrow M_{k}$ is a covering map, and so is $q_{k}: Z^{(k)} \rightarrow Z_{k}$, so $\mathcal{O}_{k}$ and $Z_{k}$ are manifolds (of dimension $n+2$ and $n+1$ ).

If $(x, t) \in P$ then

$$
\gamma_{k}:[0, t / k] \rightarrow Z_{k}, \quad \tau \mapsto\left(q_{k} g_{k}\left(\phi_{\tau} x, t\right), t\right)
$$

is a nonbounding 1 -cycle in $Z_{k}$. This is proven in [3].

\section{Definition of the index}

We retain the notation adopted above. We first consider the case where $Z_{k}$ is orientable. We call this the "orientable case". Choose a generator $1 \in$ $\bar{H}^{n+1}\left(Z_{k} \times Z_{k}, Z_{k} \times Z_{k} \backslash \Delta\right.$ ) (of course, we need not worry about singular or Alexander-Spanier cohomology at this point). Then we map

$$
\bar{H}^{n+1}\left(Z_{k} \times Z_{k}, Z_{k} \times Z_{k} \backslash \Delta\right) \stackrel{\eta^{*}}{\longrightarrow} \bar{H}^{n+1}\left(\mathcal{O}_{k}, \mathcal{O}_{k} \backslash P_{k}\right)
$$

where $P_{k}:=\left\{(\xi, s) \in \mathcal{O}_{k} \mid \psi_{s}^{k} \xi=\xi\right\}$ and $\eta: \mathcal{O}_{k} \rightarrow Z_{k} \times Z_{k}$ is defined by $\eta(\xi, s)=\left(\xi, \psi_{s}^{k} \xi\right)$. By Poincaré duality $\left(\left[9\right.\right.$, p. 363]) $\bar{H}^{n+1}\left(\mathcal{O}_{k}, \mathcal{O}_{k} \backslash P_{k}\right) \cong \bar{H}_{1} P_{k}$. So we arrive at an element $J_{k}^{\prime} \in \bar{H}_{1} P_{k}$. 
We now proceed to define a homomorphism $S: \bar{H}_{1} P_{k} \rightarrow H_{1}^{s}\left(P_{k} \times S^{1} ; \mathbb{Z}_{k}\right)$. We decompose $P_{k}$ into finitely many disjoint open (hence also closed) sets $P_{k}^{1}, \ldots, P_{k}^{l}$ such that all periodic orbits in $P_{k}^{j}$ are homotopic in $\mathcal{O}_{k}$ (more precisely, if $\left(\xi_{1}, t_{1}\right),\left(\xi_{2}, t_{2}\right) \in P_{k}^{j}$ we require that the mappings $\left[0, t_{j}\right] \rightarrow \mathcal{O}_{k}, s \mapsto\left(\psi_{s}^{k} \xi_{j}, t_{j}\right)$ be homotopic).

Since the $P_{k}^{j}$ are disjoint compact subsets of $\mathcal{O}_{k}$ there are disjoint neighbourhoods $\Pi_{k}^{j} \supset P_{k}^{j}$ in $\mathcal{O}_{k}$. We decompose each $\Pi_{k}^{j}$ into its connected components (which are open and path-connected) and split the $P_{k}^{j}$ accordingly. So by slightly changing the notation we have that $P_{k}$ decomposes into $P_{k}^{1}, \ldots, P_{k}^{r}$ and each $P_{k}^{j}$ is contained in an open path-connected neighbourhood $\Pi_{k}^{j}$. Now by [9, p. 244, (8.12)] we have that $\bar{H}^{*} P_{k} \cong \bigoplus_{j=1}^{r} \bar{H}^{*} P_{k}^{j}$ so it will be sufficient to describe $S_{j}: \bar{H}_{1} P_{k}^{j} \rightarrow H_{1}^{s}\left(P_{k} \times S^{1} ; \mathbb{Z}_{k}\right)$.

Call $i_{j}: P_{k}^{j} \rightarrow \Pi_{k}^{j}$ the inclusion, so $i_{j_{*}}: \bar{H}_{1} P_{k}^{j} \rightarrow H_{1} \Pi_{k}^{j}$. By the Künneth theorem we have that

$$
H_{1}^{s}\left(P_{k}^{j} \times S^{1} ; \mathbb{Z}_{k}\right) \cong\left(H_{0}^{s}\left(P_{k}^{j} ; \mathbb{Z}_{k}\right) \otimes H_{1} S^{1}\right) \oplus\left(H_{1}^{s}\left(P_{k}^{j} ; \mathbb{Z}_{k}\right) \otimes H_{0} S^{1}\right)
$$

since the Tor-terms vanish. So it will be sufficient to describe a homomorphism $H_{1} \Pi_{k}^{j} \rightarrow H_{0}^{s}\left(P_{k}^{j} ; \mathbb{Z}_{k}\right)$.

Choose a periodic point $\left(\xi_{j}, t_{j}\right) \in P_{k}^{j}$. By [8, p. 71] we may compute $H_{1}^{s} \Pi_{k}^{j}$ using only singular simplices $\sigma:[0,1] \rightarrow \Pi_{k}^{j}$ with $\sigma(0)=\sigma(1)=\left(\xi_{j}, t_{j}\right)$.

Let $\alpha \in H_{1}^{s} \Pi_{k}^{j}$. Choose a cycle $c=\sum_{i=1}^{\nu} \lambda_{i} \sigma_{i}$ representing $\alpha$ with singular 1 -simplices $\sigma_{i}:[0,1] \rightarrow \Pi_{k}^{j}$ which have 0 and 1 mapped to $\left(\xi_{j}, t_{j}\right)$. We choose a point $\left(\widetilde{\xi}_{j}, t_{j}\right)$ with $q_{k}\left(\widetilde{\xi}_{j}\right)=\xi_{j}$ and lift the loop $\sigma_{i}$ to $\left(\widetilde{\xi}_{j}, t_{j}\right)$. Then there is a $\mu_{i} \in \mathbb{Z}_{k}$ such that the endpoint of the lifted path equals $\zeta_{1}^{\mu_{i}} \cdot\left(\widetilde{\xi}_{j}, t_{j}\right)$ where $\zeta_{1}$ is the covering transformation defined by $\left(\widetilde{\xi}_{1}, \ldots, \widetilde{\xi}_{k}\right) \mapsto\left(\widetilde{\xi}_{2}, \ldots, \widetilde{\xi}_{k}, \widetilde{\xi}_{1}\right)$. Let $S_{0}(\alpha):=\sum_{i=1}^{\nu} \lambda_{i} \mu_{i}$ (considered as an element of $\mathbb{Z}_{k}$ ). Then we map $\alpha$ to $S(\alpha):=$ $S_{0}(\alpha)\left[\left(\xi_{j}, t_{j}\right)\right]$ where $\left[\left(\xi_{j}, t_{j}\right)\right]$ is the singular 0 -cycle in $H_{0}^{s}\left(P_{k}^{j} ; \mathbb{Z}_{k}\right)$ defined by the point $\left(\xi_{j}, t_{j}\right)$.

If we choose another cycle $c^{\prime}$ representing $\alpha$ we have that $c-c^{\prime}$ is a boundary, so the corresponding loops lie in the commutator subgroup of $\pi_{1}\left(\Pi_{k}^{j} ;\left(\xi_{j}, t_{j}\right)\right)$. But if we lift a commutator $\alpha * \beta * \overleftarrow{\alpha} * \overleftarrow{\beta}$ to $\left(\widetilde{\xi}_{j}, t_{j}\right)$ we get a closed loop, so the corresponding coefficient $\mu$ vanishes (here, by $\overleftarrow{\alpha}$ we denote of course the loop $t \mapsto \alpha(1-t))$. Since $\Pi_{k}^{j}$ is path-connected choosing a different base point will not change the result either.

We define a mapping $T: H_{0}^{s}\left(P_{k}^{j} ; \mathbb{Z}_{k}\right) \rightarrow H_{1}^{s}\left(P_{k} ; \mathbb{Z}_{k}\right)$ by mapping a 0 -cycle $[(\xi, t)]$ to the singular simplex $[0,1] \rightarrow P_{k}$ given by $s \mapsto\left(\psi_{t s}^{k} \xi, t\right)$. So we now arrive at an element $T S\left(J_{k}^{\prime}\right)$. Applying the inclusion $j_{k}: P_{k} \rightarrow \mathcal{O}_{k}$ we find $J_{k}:=$ $j_{k_{*}} T S\left(J_{k}^{\prime}\right)$.

From our construction, it is obvious that there are periodic orbits $\gamma_{1}, \ldots, \gamma_{r}$ $\left(\gamma_{j}:\left[0, t_{j}\right] \rightarrow \mathcal{O}_{k}\right.$ with $\gamma_{j}(s)=\left(\psi_{s}^{k} \xi, t_{j}\right)$ with $\xi$ a periodic point $)$ such that $J_{k}=$ 
$\sum_{i=1}^{r} c_{i}\left[\gamma_{i}\right]$ and we let $i_{k}(X, \Phi, \Omega):=\sum_{i=1}^{r} c_{i} / m_{i}^{2}$ where $m_{i}$ is the multiplicity of $\gamma_{i}$. It is obvious that the index satisfies the additivity property (A).

As to $(\mathrm{H})$ we know from [2] that the homological index is homotopy invariant, so the only point to be checked is the mapping $S$. Let $\Omega$ be open in $M \times(0, \infty) \times$ $[0,1]$ and let $\Phi: \bar{\Omega} \rightarrow M$ be as in $(\mathrm{H})$. Then we have a local semiflow $\Phi^{\prime}$ on $M \times[0,1]$ defined by $(x, \lambda), t \mapsto\left(\Phi^{\lambda}(x, t), \lambda\right)$. Form $M^{(k)} \times[0,1]$, let

$$
g_{k}((x, \lambda), t)=\left(x, \Phi^{\lambda}(x, t / k), \ldots, \Phi^{\lambda}(x,(k-1) t / k), \lambda\right),
$$

find a set $\Omega^{\prime \prime} \subset M \times(0, \infty) \times[0,1]$ as in the Lemma, form $\mathcal{O}_{k}, Z^{(k)}$, and $Z_{k}$ as above where we always use $\lambda$ as the last coordinate. $\Phi^{\prime}$ then gives rise to a local semiflow $\Psi$ on $Z_{k}$ and we may arrange things in such a way that the corresponding $\Psi^{\lambda}$ is just $\Psi$ restricted to the set $Z_{k}^{\lambda}$ of points in $Z_{k}$ with last coordinate $\lambda$. We have again a local semiflow $\Psi^{k}$ on $Z_{k}$.

Decompose the set of periodic points $P_{k}$ of $\Psi^{k}$ as above so that we have path-connected sets $\Pi_{k}^{j} \supset P_{k}^{j}$. Let $\lambda_{0} \in[0,1]$. Then $P_{k}^{j} \cap Z_{k}^{\lambda}$ and $\Pi_{k}^{j} \cap Z_{k}^{\lambda}$ may serve as the corresponding sets for $\Psi^{\lambda}$. If $\alpha \in H_{1}^{s}\left(\Pi_{k}^{j} \cap Z_{k}^{\lambda_{0}}\right)$ is represented by $\sum_{i=1}^{\nu} \lambda_{i} \sigma_{i}$, then $\sigma_{i}$ is of the form $\sigma_{i}^{\prime} \times\left\{\lambda_{0}\right\}$. So for $\lambda$ sufficiently close to $\lambda_{0}$, $\sigma_{i}^{\prime} \times\{\lambda\}$ will be defined and homotopic to $\sigma_{i}$. But it is obvious that $\sigma_{i}^{\prime} \times\{\lambda\}$ gives rise to the same $\mu_{i}$ as $\sigma_{i}$.

Proposition. The index $i_{k}$ satisfies the normalization property in the orientable case.

Proof. The proof is split into several steps. We start by showing that $I(M, \Phi, \Omega)=\iota[\gamma]$ if $M$ is an orientable manifold and $P$ consists of a single periodic orbit $\gamma$. If $m$ is the multiplicity of $\gamma$ this means that $i_{k}(M, \Phi, \Omega)=\iota / m$. If $Z_{k}$ is orientable we may apply this result to $I\left(Z_{k}, \Psi^{k}, \mathcal{O}_{k}\right)$. So in the last step we have to compare the fixed point indices of the Poincaré mappings for $\Phi$ and $\Psi^{k}$.

Assuming for the moment that $I(M, \Phi, \Omega)$ is a multiple of $[\gamma]$ we see that we will not need the homomorphisms $S$ and $T$ in this case since applying the result to $Z_{k}$ we will have that $T S\left(\left[\gamma_{k}\right]\right)=m\left[\gamma_{k}\right]$ (where $\gamma_{k}: s \mapsto\left(q_{k} g_{k}\left(\phi_{s} x, t_{0}\right), t_{0}\right)$ for some point $x \in|\gamma|$ and $t_{0}=m p(\gamma)$ ). The factor $m$ then cancels since in the definition of $i_{k}$ we divide by $m^{2}$.

We now turn to the verification of (N). Since the argument in [2] is somewhat sketchy we give a detailed proof.

With the notation from $(\mathrm{N})$ we assume that $X$ is an $n$-dimensional orientable manifold. Observe that the definition of $S$ was the only place where we needed coefficients in $\mathbb{Z}_{k}$. So we now show that the normalization property holds in case of integer coefficients.

Let $\gamma^{\prime}:[0, p(\gamma)] \rightarrow X$ be defined by $s \mapsto \phi_{s} x$ where $x$ is a point on $|\gamma|$. We choose a neighbourhood $\Omega_{0}$ of $\left|\gamma^{\prime}\right|$ in $\Omega \cap\left(X \times\left\{t_{0}\right\}\right)$ and an $\varepsilon>0$ such that 
$V:=\Omega_{0} \times\left(t_{0}-\varepsilon, t_{0}+\varepsilon\right) \subset \Omega$. We choose a section $\Sigma$ at $x$, and we choose $\Omega_{0}$ and $\varepsilon$ so small that $\tau_{m}$ is defined on $\Sigma$ and that $\eta$ is defined on $V \backslash \Sigma$.

Let $\tau_{-}:=\inf \{\tau(y) \mid y \in \Sigma\}$ and $\tau_{+}:=\sup \{\tau(y) \mid y \in \Sigma\}$. We now claim that we may choose $\Omega_{0}$ so small that $\left|\gamma^{\prime}\right|$ is a deformation retract of $\Omega_{0}$ (hence also of $V$ ). Of course, this need not be true for an arbitrary embedded circle, but we have a more favourable situation: For $t \in\left(0, \tau_{+}\right)$let $\Sigma_{t}:=\left\{y \in \Omega_{0} \backslash \Sigma \mid \eta(y)=t\right\}$ and $\Sigma_{0}:=\Sigma$. Then $\bigcup_{t \in\left[0, \tau_{+}\right)} \Sigma_{t}$ contains a neighbourhood $W$ of $\left|\gamma^{\prime}\right|$. (By reparametrizing $\Phi$ in a neighbourhood of $\left|\gamma^{\prime}\right|$ we might even arrange things in such a way that $\tau_{-}=\tau_{+}=p(\gamma)$ so that each point in neighbourhood of $\left|\gamma^{\prime}\right|$ is in a unique $\Sigma_{t}$ and $\Sigma_{t} \cap\left|\gamma^{\prime}\right|=\left\{\phi_{p-t} x\right\}$.)

Reducing the size of $W$ if necessary, we may assume that $W \subset \Omega_{0}$. By what we have just seen we may cover $\left|\gamma^{\prime}\right|$ by coordinate neighbourhoods $U_{r}=$ $U_{0}, \ldots, U_{r-1}$ contained in $W$ with charts $u_{r}=u_{0}, \ldots, u_{r-1}$ such that $U_{i} \cap U_{j} \neq \emptyset$ for $i, j \in\{0, \ldots, r\}$ if and only if $|i-j| \leq 1$ and such that each $u_{i}\left(U_{i}\right)$ is an open ball in $\mathbb{R}^{n}$. We choose a partition of unity $\lambda_{0}, \ldots, \lambda_{r-1}$ subordinate to $U_{0}, \ldots, U_{r-1}$ and we let again $\lambda_{r}:=\lambda_{0}$ and we choose retractions $r_{i}: U_{i} \rightarrow U_{i} \cap$ $\left|\gamma^{\prime}\right|$. Then we define $h_{i}: U_{i} \times[0,1] \rightarrow U_{i}$ by $h_{i}(y, t)=u_{i}^{-1}\left((1-t) u_{i}(y)+t u_{i}\left(r_{i}(y)\right)\right)$.

Now we are going to paste the $h_{i}$ together to obtain the deformation. Choose a metric $d$ for $X$ and let $y \in U_{i} \cap U_{i+1}$ and $t \in[0,1]$. Choosing the covering fine enough we may assume that either $r_{i+1}(y)=\phi_{\sigma} r_{i}(y)$ or $r_{i}(y)=\phi_{\sigma} r_{i+1}(y)$ with $0 \leq \sigma<p / 4$.

Let us assume that the first case occurs. Let then

$$
D=d\left(h_{i}(y, t), r_{i}(y)\right)+d\left(r_{i}(y), r_{i+1}(y)\right)+d\left(r_{i+1}(y), h_{i+1}(y, t)\right) .
$$

If $\alpha:=d\left(h_{i}(y, t), r_{i}(y)\right)>0$ and $0 \leq s \leq \alpha / D$ we let

$$
k(y, t, s)=u_{i}^{-1}\left((1-D s / \alpha) u_{i}\left(h_{i}(y, t)\right)+(D s / \alpha) u_{i}\left(r_{i}(y)\right)\right) .
$$

If $\alpha=0$ we let $k(y, t, 0)=h_{i}(y, t)=r_{i}(y)$. If $\beta:=d\left(r_{i}(y), r_{i+1}(y)\right)>0$ we let $k(y, t, s)=\Phi\left(r_{i}(y), \sigma(D s-\alpha) / \beta\right)$ if $\alpha / D \leq s \leq(\alpha+\beta) / D$.

Finally, if $\gamma:=D-\alpha-\beta>0$, we let

$$
k(y, t, s)=u_{i+1}^{-1}\left((1-s) D u_{i+1}\left(r_{i+1}(y)\right) / \gamma+(D s-\alpha-\beta) u_{i+1}\left(h_{i+1}(y, t)\right) / \gamma\right)
$$

if $(\alpha+\beta) / D \leq s \leq 1$. Then we put $H(y, t):=k\left(y, t, \lambda_{i+1}(y)\right)$. Of course, we let $H(y, t)=h_{i}(y, t)$ if $y \in U_{i} \backslash\left(U_{i+1} \cup U_{i-1}\right)$. This yields the required deformation. So we are done if we let $\Omega_{0}:=\bigcup_{i=0}^{r-1} U_{i}$.

Now we choose an open set $B_{0} \ni x$ in $\Omega_{0}$ which is homeomorphic to an open ball. For $(y, t) \in B:=B_{0} \times\left(t_{0}-\varepsilon / 2, t_{0}+\varepsilon / 2\right)$ we let

$$
\tau_{m}^{\prime}(y, t)=t+\tau_{m}(y)-t_{0}+2\left(t_{0}-\tau_{m}(y)\right)\left(t-t_{0}\right) / \varepsilon
$$


if $t_{0} \leq t \leq t_{0}+\varepsilon / 2$ and, if $t_{0}-\varepsilon / 2 \leq t \leq t_{0}$,

$$
\tau_{m}^{\prime}(y, t)=t+\tau_{m}(y)-t_{0}+2\left(\tau_{m}(y)-t_{0}\right)\left(t-t_{0}\right) / \varepsilon .
$$

If $\varepsilon \geq\left|t-t_{0}\right| \geq \varepsilon / 2$ we let $\tau_{m}^{\prime}(y, t)=t$. Then we choose $0<t_{1}<t_{2}$ such that $\eta(y) \geq p(x)-t_{2}$ or $\eta(y)<t_{2}$ implies $y \in B_{0}$.

For $(y, t) \in B$ we abbreviate $t^{\prime}:=\left(t-\tau_{m}^{\prime}(y, t)\right) /\left(t_{2}-t_{1}\right)$ and we let

$$
F(y, t)= \begin{cases}\phi_{t} y & \text { if } t_{2} \leq \eta(y) \leq p(x)-t_{2}, \\ \Phi\left(y,-t^{\prime} \eta(y)+t+t^{\prime}\left(p(x)-t_{2}\right)\right) & \text { if } p(x)-t_{2} \leq \eta(y) \leq p(x)-t_{1}, \\ \Phi\left(y, t^{\prime} \eta(y)+t-t^{\prime} t_{2}\right) & \text { if } t_{1} \leq \eta(y) \leq t_{2}, \\ \Phi\left(y, \tau_{m}^{\prime}(y)\right) & \text { if } \eta(y) \geq p(x)-t_{1} \text { or } \eta(y) \leq t_{1} .\end{cases}
$$

Note that $F(y, t)$ is on the same flowline as $\phi_{t} y$, so $Q:=\{(y, t) \in V \mid F(y, t)=t\}$ has points only on $\left|\gamma^{\prime}\right| \times\left(t_{0}-\varepsilon, t_{0}+\varepsilon\right)$.

Now for $\left|t-t_{0}\right| \geq \varepsilon / 2$ we have that $\tau_{m}^{\prime}(y, t)=t$, so fixed points of $F$ have to lie on $\left|\gamma^{\prime}\right| \times\left(t_{0}-\varepsilon / 2, t_{0}+\varepsilon / 2\right)$. $F(x, t)$ is constructed in such a way that $F\left(\cdot, t_{0}\right)$ equals $\pi$ in a neighbourhood of $S$ and $\phi_{t_{0}}$ outside a larger neighbourhood. Moreover, it is easy to see that $Q$ is homeomorphic to $\left|\gamma^{\prime}\right|$ (since we just made an affine change in the time-parameter). We choose a neighbourhood $\mathcal{O}$ of $x$ such that $\mathcal{O} \subset B_{0}, \mathcal{O}$ is homeomorphic to an open ball and $F\left(\cdot, t_{0}\right)=\pi$ in $\mathcal{O}$. So we have $I(X, \Phi, V)=I(X, F, V)$ (note that $(\mathrm{H})$ requires a deformation through semiflows, but our definition works for arbitrary deformations).

Let $I(X, \Phi, V)=I(X, F, V)=I\left[\gamma^{\prime}\right]$. Call $c$ the generator of $\bar{H}^{n}(V, V \backslash Q)$ which is mapped to $\left[\gamma^{\prime}\right]$ under the Poincaré isomorphism $\bar{H}^{n}(V, V \backslash Q) \cong \bar{H}_{1} Q \cong$ $\mathbb{Z}$. So the diagonal class $\tau \in H^{n}(X \times X, X \times X \backslash \Delta)$ is mapped to $I c$ by the mapping induced by $(x, t) \mapsto(F(x, t), t)$.

If we define $i: \mathcal{O} \rightarrow V$ with $i(y)=\left(y, t_{0}\right)$, we have that $i^{*}$ maps the generator $c$ of $\bar{H}^{n}(V, V \backslash Q)$ to a generator of $\bar{H}^{n}(\mathcal{O}, \mathcal{O} \backslash\{x\}) \cong \mathbb{Z}$. This is seen as follows: For $n>2$ we have that

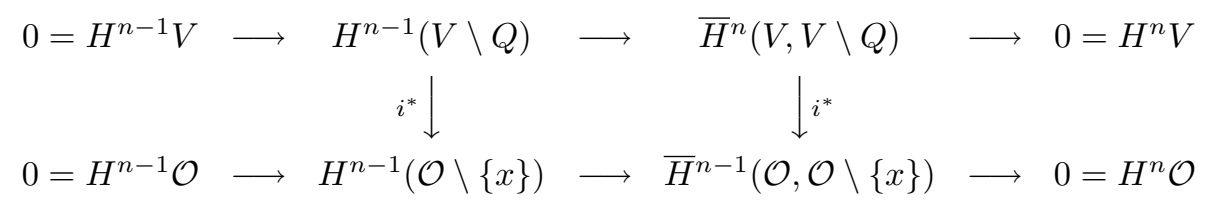

Here, the nonzero terms in the horizontal rows are isomorphic (and isomorphic to $\mathbb{Z}$ ), so we will be done if we can show that $H^{n-1}(V \backslash Q) \rightarrow H^{n-1} S^{n-1} \cong$ $H^{n-1}(\mathcal{O} \backslash\{x\})$ is an isomorphism. But this is obvious since the inclusion maps $S^{n-1}$ into $\Omega_{0} \times\left\{t_{0}\right\}$ and $Q \cap\left(\mathcal{O} \times\left\{t_{0}\right\}\right)=\left\{\left(x_{0}, t_{0}\right)\right\}$. (The case $n=1$ is uninteresting, and $n=2$ is either treated similarly or we just add an extra dimension.)

Now ind $(X, \pi, \mathcal{O})$ is the image of $\tau$ under $g:(\mathcal{O}, \mathcal{O} \backslash\{x\}) \rightarrow(X \times X, X \times X \backslash \Delta)$ where $g(y)=(y, \pi(y))=\left(y, F\left(y, t_{0}\right)\right)$, i.e., $g^{*} \tau=\operatorname{ind}(X, \pi, \mathcal{O}) \cdot i^{*} c$ which proves our assertion. 
So in our situation we have that $I\left(Z_{k}, \Psi^{k}, \mathcal{O}_{k}\right)=\left(\iota_{k}^{\prime} / m\right)\left[\gamma_{k}\right]$ where $\iota_{k}^{\prime}$ is the fixed point index of the Poincaré mapping of $\gamma_{k}: s \mapsto\left(q_{k} g_{k}\left(\phi_{s} x, t\right), t\right)$ (where $x$ is a point on $\gamma$ and $t_{0}=m p(\gamma)$ ). (Note that as opposed to [2] we here have to divide by the multiplicity since we do not assume that $t_{0}$ equals the minimal period of $\gamma$.)

On the other hand, by [3, Proposition 2] we have that $\iota_{k}^{\prime}$ equals the fixed point index $\iota$ of the Poincaré mapping corresponding to $\gamma$. Since the situation in [3] is somewhat different we sketch the argument which is based on the commutativity property of the fixed point index: Since $\gamma$ is isolated in $X$ we have that $\gamma_{k}$ is isolated in $Z_{k}$.

Choose an $\varepsilon>0$ and a neighbourhood $V$ of $|\gamma|$ such that $V \times\left(t_{0}-\varepsilon, t_{0}+\varepsilon\right) \subset$ $\Omega^{\prime \prime}$. Let

$$
W:=\left\{\left(q_{k} g_{k}(x, t,), t, s\right)|x \in V,| t-t_{0}|<\varepsilon,| s-t / k \mid<\delta\right\}
$$

where $\delta$ is taken from (iii) in the Lemma. On $W \times[0,1]$ define

$$
\Theta\left(q_{k} g_{k}(x, t), t, s, \sigma\right):=\left(q_{k} g_{k}\left(\phi_{s} x,(1-\sigma) t+\sigma t_{0}\right),(1-\sigma) t+\sigma t_{0}\right) .
$$

Thus, on $W, \Theta$ defines a homotopy between $\Psi^{k}$ and $\Psi^{\prime}$ defined by

$$
\psi_{s}^{\prime}\left(q_{k} g_{k}(x, t,), t\right)=\left(q_{k} i_{k} \phi_{s} x, t_{0}\right)
$$

where $i_{k}(x):=g_{k}\left(x, t_{0}\right)$. Since $\Psi^{\prime}$ maps into the ANR $V_{k}:=q_{k} g_{k}(V)$ the commutativity property of the fixed point index shows that in computing the fixed point index of the Poincaré map for $\Psi^{\prime}$ we may restrict $\Psi^{\prime}$ to $V_{k}$ and compute the fixed point index with respect to the ANR $V_{k}$. So we have a local semiflow $\Psi^{\prime \prime}$ on $V_{k}$ given by $\psi_{s}{ }^{\prime \prime} q_{k} i_{k} x=q_{k} i_{k} \phi_{s} x$, and we have to show that $\iota$ equals the fixed point index $\iota_{k}^{\prime}$ of the Poincaré mapping for $\Psi^{\prime \prime}$ for the period $t_{0} / k$ at $q_{k} i_{k} x_{0}$.

We now choose a neighbourhood $B$ of $x_{0}$ in $V$ such that the sets $\Phi(B \times$ $\left.\left\{j t_{0} / k\right\}\right)$ are disjoint for $j=0, \ldots, k-1$ and such that $q_{k} i_{k}(B)$ is contained in a neighbourhood $N$ of $q_{k} i_{k} x_{0}$ in $X_{k}$ which is evenly covered by $q_{k}$.

Choose a neighbourhood $N_{1}$ of $i_{k} x_{0}$ in $X^{(k)}$ which is mapped homeomorphically onto $N$ by $q_{k}$ and call $\ell:=\left(q_{k} \mid N_{k}\right)^{-1} \mid V: N \cap V_{k} \rightarrow N_{1}$. We now choose a neighbourhood $\mathcal{O}$ of $x_{0}$ in $B$ and a section $\Sigma$ such that the Poincaré mapping $\pi$ for the period $t_{0}$ at $x_{0}$ is defined. Moreover, we choose the section $\Sigma$ so small that $\eta\left(\phi_{(k-1) t_{0} / k} y\right)<\ldots<\eta\left(\phi_{t_{0} / k} y\right)<\tau(y)$ whenever $y \in \Sigma$. Call $\operatorname{pr}_{1}: X^{(k)} \rightarrow X$ the projection onto the first factor. If $m:=t_{0} / p\left(x_{0}\right)=1$ we let $\pi^{\prime}:=\pi$, and if $m>1$ we denote by $\pi^{\prime}$ the Poincaré mapping for the period $(m-1) p\left(x_{0}\right)$.

We now claim that $\iota$ equals $\operatorname{ind}\left(X, \operatorname{pr}_{1} \ell q_{k} i_{k} \phi_{t_{0} / k} \pi^{\prime}, \mathcal{O}\right)$. In fact, let $y \in \mathcal{O}$. Then $q_{k} i_{k} \phi_{t_{0} / k} \pi^{\prime} y$ is the equivalence class of $\left(\phi_{t_{0} / k} \pi^{\prime} y, \ldots, \phi_{(k-1) t_{0} / k} \pi^{\prime} y, \phi_{t_{0}} \pi^{\prime} y\right)$ which is lifted by $\ell$ to $\left(\phi_{t_{0}} \pi^{\prime} y, \phi_{t_{0} / k} \pi^{\prime} y, \ldots, \phi_{(k-1) t_{0} / k} \pi^{\prime} y\right)$. But this means that $\operatorname{pr}_{1} \ell q_{k} i_{k} \phi_{t_{0} / k} \pi^{\prime}=\phi_{t_{0}} \pi^{\prime}$. 
Now consider the homotopy $\mathrm{cl} \mathcal{O} \times[0,1] \rightarrow X$ which is given by $(y, s) \mapsto$ $\Phi\left(\pi^{\prime} y, s \tau\left(\pi^{\prime} y\right)+(1-s) t_{0}\right)$. During the homotopy, each point stays on its flowline and the only fixed point is $x_{0}$. For $s=0$, the homotopy starts with $\phi_{t_{0}} \pi^{\prime}=\operatorname{pr}_{1} \ell q_{k} i_{k} \phi_{t_{0} / k} \pi^{\prime}$ and it ends, for $s=1$, at $\pi$. So we see that $\iota=$ $\operatorname{ind}\left(X, \operatorname{pr}_{1} \ell q_{k} i_{k} \phi_{t_{0} / k} \pi^{\prime}, \mathcal{O}\right)$.

The commutativity property for the fixed point index now shows that

$$
\operatorname{ind}\left(X, \operatorname{pr}_{1} \ell q_{k} i_{k} \phi_{t_{0} / k} \pi^{\prime}, \mathcal{O}\right)=\operatorname{ind}\left(V_{k}, q_{k} i_{k} \phi_{t_{0} / k} \pi^{\prime} \operatorname{pr}_{1} \ell, q_{k} i_{k}(\mathcal{O})\right) .
$$

So it remains for us to show that the righthand term equals $\iota_{k}^{\prime}$.

Denote by $\Sigma^{\prime}$ the set of those $i_{k} x$ such that $\phi_{j / k t_{0}} x \in \Sigma$ for some $j \in$ $\{0, \ldots, k-1\}$ and let $\Sigma_{k}:=q_{k}\left(\Sigma^{\prime}\right)$. Let then $q_{k} i_{k} y \in \Sigma_{k}$ and assume that $\phi_{j t_{0} / k} y \in \Sigma$. Define

$$
\begin{array}{ll}
\tau^{\prime}\left(q_{k} i_{k} y\right):=\eta\left(\phi_{(j-1) t_{0} / k} y\right) & \text { if } j>0, \\
\tau^{\prime}\left(q_{k} i_{k} y\right):=\eta\left(\phi_{(k-1) t_{0} / k} y\right) & \text { if } j=0 .
\end{array}
$$

It is easy to see that $\tau^{\prime}$ is continuous and that $\Sigma_{k}$ is a section for $\Psi^{\prime}$ at $q_{k} i_{k} x_{0}$. So we consider the homotopy $\operatorname{cl} q_{k} i_{k}(\mathcal{O}) \times[0,1] \rightarrow V_{k}$ which is given by

$$
(\xi, s) \mapsto q_{k} i_{k} \Phi\left(\pi^{\prime} \operatorname{pr}_{1} \ell \xi,(1-s) t_{0} / k+s \eta\left(\phi_{(k-1) t_{0} / k}\left(\pi^{\prime} \ell \xi\right)\right) .\right.
$$

Again, during this homotopy, each point stays on its flowline under $\Psi^{\prime \prime}$ and the only fixed point is $q_{k} i_{k} x_{0}$. The homotopy starts, for $s=0$, with $q_{k} i_{k} \phi_{t_{0} / k} \pi^{\prime} \operatorname{pr}_{1} \ell$ and it ends, for $s=1$, at the Poincaré mapping for $\Psi^{\prime \prime}$ and the period $t_{0} / k$. So we see that

$$
\iota_{k}^{\prime}=\operatorname{ind}\left(V_{k}, q_{k} i_{k} \phi_{t_{0} / k} \pi^{\prime} \operatorname{pr}_{1} \ell, q_{k} i_{k}(\mathcal{O})\right) .
$$

It is, however, obvious, that $\iota_{k}$ can be obtained by computing the fixed point index of the Poincaré mapping using $\mathbb{Z}_{k}$-coefficients. (If $\operatorname{dim} X=n, D \subset X$ is an open set and $f: \bar{D} \rightarrow X$ is continuous with no fixed points on $\partial D$ the fixed point index in $\mathbb{Z}_{k}$ is defined to be $\left\langle g^{*} \tau_{k}, \mu_{k}\right\rangle$ where $\tau_{k} \in H^{n}\left(D \times D, D \times D \backslash \Delta ; \mathbb{Z}_{k}\right)$ is the diagonal class, $F$ is the fixed point set of $f$ in $D, \mu_{k} \in H_{n}\left(D, D \backslash F\right.$; $\left.\mathbb{Z}_{k}\right)$ is the fundamental class and $g(x)=(x, f(x))$.)

\section{The index for local semiflows on simplicial complexes and the nonorientable case}

Now we turn to the case where $X$ is a finite simplicial complex. We embed $X$ into some $\mathbb{R}^{n}$ and we choose an open set $\mathcal{O} \supset X$ and a retraction $r: \mathcal{O} \rightarrow X$.

Let $R: \mathcal{O} \times \mathbb{R} \rightarrow X \times \mathbb{R}$ be defined by $R:=r \times$ id and let $\widetilde{\Omega}:=R^{-1}(\Omega)$. We define $\Psi: \widetilde{\Omega} \rightarrow X$ by $\Psi(x, t)=\Phi(r(x), t)$. Then we have

$$
\{(x, t) \in \widetilde{\Omega} \mid \Psi(x, t)=x\}=\{(x, t) \in \Omega \mid \Phi(x, t)=x\}
$$

and we may repeat the above construction for $\Psi$. 
There is but one problem: we have to check that the definition does neither depend on the embedding nor on the retraction. This is, however, shown in [2] where it is proved that the homological index does not depend on either of these choices. If $X$ is an infinite simplicial complex, by (H2) we have that $\operatorname{cl} \Phi(\Omega)$ is a compact subset of $X$ hence contained in a finite subcomplex $K$, so we may define the index by restricting $\Phi$ to $K$.

Now we return to the case where $M$ is a manifold, $\Omega$ is open in $M \times(0, \infty)$ and $\Phi$ is a local semiflow satisfying (H1) and (H2). We proceed exactly as in the manifold case and form the (possibly non-orientable) manifold $Z_{k}$, the open set $\mathcal{O}_{k}$, and the local semiflow $\Psi^{k}$. Then we embed $Z_{k}$ as a neighbourhood retract in some $\mathbb{R}^{n}$, choose a neighbourhood $U \supset Z_{k}$ in $\mathbb{R}^{n}$ and a retraction $r: U \rightarrow Z_{k}$.

We define $R: U \times \mathbb{R} \rightarrow Z_{k} \times \mathbb{R}$ by $R(x, t)=(r(x), t)$ and let $V:=R^{-1}\left(\mathcal{O}_{k}\right)$. We define $\Psi^{\prime}: V \rightarrow Z_{k}$ by $\Psi^{\prime}(x, t)=\Psi^{k}(r(x), t)$. Then $P^{\prime}:=\{(x, t) \in V \mid$ $\left.\Psi^{\prime}(x, t)=x\right\}$ is a subset of $P_{k}$ and we may define $i_{k}(X, \Phi, \Omega)=i_{k}\left(\mathbb{R}^{n+1}, \Psi^{\prime}, V\right)$.

The proof that this definition depends neither on the choice of embedding nor of retraction reduces to the observation that the homological index $I\left(Z_{k}, \Psi^{k}, \mathcal{O}_{k}\right)$ does not depend on these choices. The verification of this fact carries over almost verbatim from [2]: as a matter of fact, the argument in [2] works for arbitrary ENR (the proof assumes a PL-embedding but this fact is never used - any embedding will do).

\section{The ANR case}

In the general case, we start by choosing a compact ANR $Y$ with $\mathrm{cl} \Phi(\Omega) \subset Y$. This is achieved as follows. Embed $X$ as a closed subset in a normed linear space $E$, choose a neighbourhood $W$ of $X$ and a retraction $r: W \rightarrow X$. By [6] there is a compact ANR $C$ such that $\operatorname{cl} \Phi(\Omega) \subset C \subset W$.

Let $Y:=r(C)$. For simplicity, we now assume that $X$ itself is compact and that we have an open subset $\Omega \subset X \times(0, \infty)$ such that $\operatorname{cl} \Phi(\Omega) \subset \mathcal{D}(\Phi)$ and such that

$$
P:=\left\{(x, t) \in \bar{\Omega} \mid \phi_{t} x=x\right\} \subset \Omega .
$$

Now we intend to choose a finite open covering $\alpha$ of $X$ with nerve $N_{\alpha}$, canonical projection $p_{\alpha}: X \rightarrow N_{\alpha}$ and realization $i_{\alpha}: N_{\alpha} \rightarrow X$ and to replace $\Phi$ by $F:(x, t) \mapsto p_{\alpha}\left(\phi_{t} i_{\alpha} x\right)$. (If $U_{1}, \ldots, U_{n} \in \alpha$ and $\bigcap_{i=1}^{n} U_{i} \neq \emptyset$, we denote by $\left\langle U_{1}, \ldots, U_{n}\right\rangle_{\alpha}$ the simplex of $N_{\alpha}$ with vertices $\left\langle U_{1}\right\rangle_{\alpha}, \ldots,\left\langle U_{n}\right\rangle_{\alpha}$. A map $p: X \rightarrow N_{\alpha}$ is said to be a canonical projection if $p(x) \in\left\langle U_{1}, \ldots, U_{n}\right\rangle_{\alpha}$ whenever $x \in \bigcap_{i=1}^{n} U_{i}$.)

Note that in defining the homological index we just need a mapping (and not a semiflow), but in order to make things work we have to make sure that $g_{k}(x, t):=(F(x, 0), F(x, t / k), \ldots, F(x,(k-1) t / k))$ consists of distinct points. 
Moreover, in defining the homomorphism $S$ we need periodic orbits of $F$ which serve as singular 1-cycles.

In order to achieve the second purpose we intend to choose $p_{\alpha}$ and $i_{\alpha}$ in such a way that $i_{\alpha} p_{\alpha}=$ id on "sufficiently many" periodic orbits.

To solve the first task we choose a metric $d$ on $X$ and let

$$
\rho:=\min \left\{d\left(z, \phi_{j t / k} z\right) \mid(z, t) \in \bar{\Omega}, j=1, \ldots, k-1\right\} .
$$

If then $F(x, i t / k)=F(x, j t / k)$ for $0 \leq i<j \leq k-1$ we would have

$$
p_{\alpha}\left(\phi_{(j-i) t / k} y\right)=p_{\alpha}(y) \quad \text { with } y=i_{\alpha}(x)
$$

which requires that $y$ and $\phi_{(j-i) t / k} y$ belong to the open star of one element of $\alpha$. So it will be sufficient to choose the covering $\alpha$ so fine that all elements have diameter less than $\rho / 3$. It is obvious that we may choose open sets $\Omega^{\prime}, \Omega^{\prime \prime}$ satisfying (i)-(iii) and (v) in the Lemma.

Now we turn to the second task and choose an open covering $\kappa$ so fine that the homological index $I\left(N_{\alpha}, F, F^{-1}(\Omega)\right)$ is defined and does neither depend on $\alpha$ nor on $p_{\alpha}$ or $i_{\alpha}$ provided the mesh of $i_{\alpha}$ is smaller than $\kappa$, cf. [2]. Moreover, we choose $\kappa$ so fine that all elements have diameter less than $\rho / 3$.

We choose an open cover $\beta$ such that each partial realization $i: K \rightarrow X$ of a finite polyhedron which has mesh $\beta$ extends to a full realization of mesh $\kappa$. This is possible since $X$ is an ANR (cf. [7, IV, Theorem 4.2]). Choose a finite open star refinement $\beta^{*}$ of $\beta$ and let $\gamma$ be a periodic orbit of $\Phi$ and let $x$ be a point on $\gamma$.

Let $\left(T^{\prime}(\gamma), T(\gamma)\right)$ be a tube around $\gamma$ with section $S(\gamma)$ at $x$. We choose a positive integer $N$ such that for $i=0, \ldots, N-1$ we have that $\operatorname{cl} \Phi(\{x\} \times$ $[i p(x) / N,(i+2) p(x) / N])$ is contained in some element of $\beta^{*}$. Then we choose a neighbourhood $W(\gamma)$ of $\gamma$ and a retraction $r_{\gamma}: W(\gamma) \rightarrow \gamma$. Let

$$
\begin{gathered}
\Gamma_{0}(x):=\Gamma_{N}(x):=\Phi(\{x\} \times((N-1) p(x) / N, p(x)] \cup[0, p(x) / N)), \\
\Gamma_{i}:=\Phi(\{x\} \times((i-1) p(x) / N,(i+1) p(x) / N) \quad \text { for } i=1, \ldots, N-1 .
\end{gathered}
$$

Then we choose $W(\gamma)$ so small that each $\Gamma_{i}^{\prime}(x):=r_{\gamma}^{-1}\left(\Gamma_{i}(x)\right)$ is contained in some element of $\beta^{*}$ and such that $\Gamma_{i}^{\prime}(x) \cap \Gamma_{j}^{\prime}(x) \neq \emptyset$ only if $|i-j| \leq 1$. Call

$$
U(\gamma):=U(x):=\bigcup_{i=0}^{N-1} \Gamma_{i}^{\prime}(x)
$$

We select periodic orbits $\gamma_{1}, \ldots, \gamma_{s}$ and points $x_{i} \in\left|\gamma_{i}\right|$ such that each periodic orbit of $\Phi$ is homotopic to one of the $\gamma_{i}$ and such that $U_{1}:=U\left(\gamma_{1}\right), \ldots, U_{s}:=$ $U\left(\gamma_{s}\right)$ cover $\operatorname{pr}_{1} P$. Reducing the size of the $U_{i}$ we may assume that $\left|\gamma_{i}\right| \cap U_{j}=\emptyset$ if $i \neq j$. Then there are neighbourhoods $U_{i}^{\prime \prime} \subset \operatorname{cl} U_{i}^{\prime \prime} \subset U_{i}^{\prime} \subset \operatorname{cl} U_{i}^{\prime} \subset U_{i}$ such that the $U_{i}^{\prime \prime}$ still cover $\operatorname{pr}_{1} P$. The set $\operatorname{pr}_{1} P$ is covered by $\alpha_{1}:=\left\{\Gamma_{i}^{\prime}\left(x_{j}\right) \mid j=\right.$ 
$1, \ldots, s, i=0, \ldots, N-1\}$. We select a finite open cover $\alpha_{2}$ of $X \backslash \bigcup_{i=1}^{s} U_{i}^{\prime}$ such that for each $V \in \alpha_{2}$ we have that

- st $_{\alpha_{2}} V$ is contained in an element $W \in \beta^{*}$ such that $W \cap \operatorname{pr}_{1} P=\emptyset$.

- If $\Omega \cap \operatorname{pr}_{1}^{-1}(V) \neq \emptyset$ then $V \cap \operatorname{pr}_{1} P=\emptyset$.

Let $\alpha$ be the covering of $X$ which consists of $\alpha_{1}$ and $\alpha_{2}$. We will now construct a canonical projection $p_{\alpha}: X \rightarrow N_{\alpha}$ which maps $\gamma_{1}, \ldots, \gamma_{s}$ homeomorphically into the one-skeleton of $N_{\alpha}$.

Let $y \in \bigcup \alpha_{1}$. Write $r_{j}(y)=\phi_{t} x_{j}$ with $0 \leq t \leq p\left(x_{j}\right)$ and $t=(\lambda+i) p\left(x_{j}\right) / N$ with $0 \leq \lambda<1,0 \leq i \leq N-1$ and let $p_{\alpha}^{\prime}(y):=(1-\lambda)\left\langle\Gamma_{i}^{\prime}\left(x_{j}\right)\right\rangle_{\alpha}+\lambda\left\langle\Gamma_{i+1}^{\prime}\left(x_{j}\right)\right\rangle_{\alpha}$.

Denote by $p_{\alpha}^{\prime \prime}: X \rightarrow N_{\alpha}$ any canonical projection and choose a continuous function $\lambda: X \rightarrow[0,1]$ such that

$$
\lambda \mid \bigcup_{i=1}^{s} U_{i}^{\prime \prime}=0 \quad \text { and } \quad \lambda \mid \bigcap_{i=1}^{s}\left(X \backslash U_{i}^{\prime}\right)=1 .
$$

If $x \in U_{j}^{\prime}$ we have that $p_{\alpha}^{\prime}(x)$ and $p_{\alpha}^{\prime \prime}(x)$ belong to a common simplex of $N_{\alpha}$, so we may define

$$
p_{\alpha}(x):=(1-\lambda(x)) p_{\alpha}^{\prime}(x)+\lambda(x) p_{\alpha}^{\prime \prime}(x) .
$$

On $\bigcap_{i=1}^{s}\left(X \backslash U_{i}^{\prime}\right)$ we let $p_{\alpha}:=p_{\alpha}^{\prime \prime}$. Obviously, on $\left|\gamma_{j}\right|, p_{\alpha}$ coincides with $p_{\alpha}^{\prime}$, so we have that $p_{\alpha}$ maps $\left|\gamma_{j}\right|$ homeomorphically onto $\left|\gamma_{j}^{\prime}\right|:=p_{\alpha}\left(\left|\gamma_{j}\right|\right)$.

On the other hand, we define now a partial realization of $N_{\alpha}$ in the following way. For $i=0, \ldots, N-1, j=1, \ldots, s$, and $0 \leq \lambda \leq 1$ we map $(1-\lambda)\left\langle\Gamma_{i}^{\prime}\left(x_{j}\right)\right\rangle_{\alpha}+$ $\lambda\left\langle\Gamma_{i+1}^{\prime}\left(x_{j}\right)\right\rangle_{\alpha}$ to $\Phi\left(x_{j},(i+\lambda) p\left(x_{j}\right) / N\right)$.

If $\langle U\rangle_{\alpha}$ is a vertex with $U \in \alpha_{2}$ we map $\langle U\rangle_{\alpha}$ to an arbitrary point of $U$. This yields a partial realization of $N_{\alpha}$ with mesh $\beta$ and we extend this map to a full realization $i_{\alpha}: N_{\alpha} \rightarrow X$ of mesh $\kappa$.

We compute now the index of the mapping $F$ on $N_{\alpha}$ as explained above where we use the one-cycles $p_{\alpha} \circ \gamma_{1}, \ldots, p_{\alpha} \circ \gamma_{s}$ and points on these orbits to represent the 0 -cycles required in the definition of the homomorphism $S$. Then we may use the argument in [2] to show the independence from the choices involved (i.e. the choice of covering, projection and realization). Properties $(\mathrm{A}),(\mathrm{H})$, and $(\mathrm{N})$ then are verified as in [2].

To sum up, we have shown

THEOREM. There exists an index for local semiflows on ANR which satisfy conditions (H'1)-(H'3) above.

As was said in the beginning I have not been able to remove the dependence from $k$ in the definition. In particular, although the index $i_{k}(X, \Phi, \Omega)$ was defined for large primes the definition would allow for products of sufficiently large primes so that one could hope to compare $i_{p}(X, \Phi, \Omega), i_{q}(X, \Phi, \Omega)$ and $i_{p q}(X, \Phi, \Omega)$. But 
$\mathbb{Z}_{p q} \cong \mathbb{Z}_{p} \oplus \mathbb{Z}_{q}$, and it is easy to see that $i_{p}(X, \Phi, \Omega)$ and $i_{q}(X, \Phi, \Omega)$ are just the two components of $i_{p q}(X, \Phi, \Omega)$ in $\mathbb{Z}_{p q}$.

\section{REFERENCES}

[1] C. C. Fenske, Periodic orbits of semiflows - Local indices and sections, Selected Topics in Operations Research and Mathematical Economics, Lecture Notes in Economics and Mathematical Systems, vol. 226, Springer, Berlin-Heidelberg-New York-Tokyo, 1984, pp. $348-360$.

[2] , A simple-minded approach to the index of periodic orbits, J. Math. Anal. Appl. 129 (1988), 517-532.

[3] _ An index for periodic orbits of functional differential equations, Math. Ann. 285 (1989), 381-392.

[4] - An index for periodic orbits of local semidynamical systems, Trans. Amer. Math. Soc. 350 (1998), 4973-4991.

[5] F. B. Fuller, An index of fixed point type for periodic orbits, Amer. J. Math. 89 (1967), 133-148.

[6] J. Girolo, Approximating compact sets in normed spaces, Pacific J. Math. 98 (1982), $81-89$.

[7] S.-T. Hu, Theory of Retracts, Wayne State University Press, Detroit, 1965.

[8] W. S. Massey, Singular Homology Theory, Springer, New York-Heidelberg-Berlin, 1980 .

[9] Homology and Cohomology Theory, Dekker, New York-Basel, 1978.

Manuscript received April 1, 2002

Manuscript revised September 14, 2002

Christian C. Fenske

Mathematisches Institut

Justus-Liebig Universität Gießen

Arndtstraße 2

D-35392 Gießen, GERMANY

E-mail address: christian.fenske@math.uni-giessen.de 